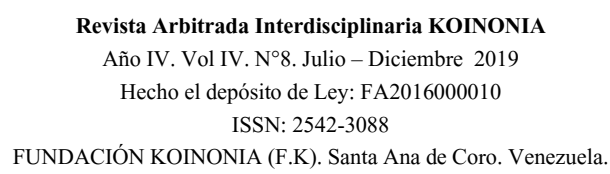

Annayeskha Grabiela González beuses

DOI: http://dx.doi.org/10.35381/r.k.v4i8.283

\title{
Incidencia de las Organizaciones Inteligentes en la Gestión del Conocimiento
}

\author{
Incidence of Intelligent Organizations in the Management of Knowledge
}

\author{
Annayeskha Grabiela González beuses \\ annayeskha@gmail.com \\ Universidad Rafael Belloso Chacin \\ Venezuela \\ https://orcid.org/0000-0002-3268-5118
}

Recibido: 30 de abril del 2019

Aprobado: 10 de junio del 2019

\section{RESUMEN}

Este artículo tiene como propósito analizar todas las aristas relacionadas con la gestión del conocimiento y su influencia en las organizaciones inteligentes, por medio de postulados teóricos de autores como Amozorrutia (2011), Salazar (2004), Arboníes (2006), entre otros. La metodología utilizada es del tipo analítica, documental, bibliográfica descriptiva, con un diseño de tipo bibliográfico no experimental. Para las unidades de análisis, se consultaron libros, artículos y otras fuentes bibliográficas. La técnica empleada para el análisis e interpretación de los datos es cualitativa de análisis crítico. Se concluyó queel conocimiento es un elemento fundamental para garantizar el éxito, cuyo fin es promover la innovación y competitividad, permitiendo que las organizaciones se mantengan en el mercado. Para ello debe contar con un líder que impulse a los colaboradores a emplear sus destrezas e intercambio de información para tener organizaciones sean inteligentes que alcancen los objetivos en el menor tiempo y costo.

Descriptores: liderazgo; gerencia; conocimiento; organización

\begin{abstract}
The purpose of this article is to analyze all the aspects related to knowledge management and its influence on intelligent organizations, through theoretical postulates of authors such as Amozorrutia (2011), Salazar (2004), Arboníes (2006), among others. The methodology used is of the analytical, documentary, descriptive bibliographic type, with a non-experimental bibliographic type design. For the units of analysis, books, articles and other bibliographic sources were consulted. The technique used for the analysis and interpretation of the data is qualitative of critical analysis. It was concluded that knowledge is a fundamental element to guarantee success, whose purpose is to
\end{abstract}


promote innovation and competitiveness, allowing the company to remain in the market. For this, it must have a leader that encourages employees to use their skills and exchange information to have smart organizations that achieve the objectives in the shortest time and cost.

Descriptors: leadership; management; knowledge; organization

\section{INTRODUCCIÓN}

Actualmente el mundo se maneja bajo escenarios cambiantes, producto de la globalización y para que las organizaciones logren subsistir en el mercado tan competitivo, es necesario que desarrollen una serie de ventajas competitivas e innoven, siendo fundamental contar con el activo intangible de mayor calidad, talento humano formado aunada a la planificación y dirección adecuada que permita a las organizaciones utilizar esas habilidades o destrezas en aras de convertirse en exitosas.

Al respecto Amozorrutia (2011), afirma que con el aprendizaje, se reconoce la importancia del capital intelectual para mantener, desarrollar y transmitir el conocimiento (tácito y/o explicito) entre los colaboradores. Por su parte Hsieh (2011), menciona que las organizaciones inteligentes es donde se aprovecha el conocimiento interno, aprendiendo y desarrollando nuevo conocimiento.

Según lo planteado por los mencionados autores, el conocimiento es un elemento clave para que la organización alcance los objetivos propuestos impulsando con ello el desarrollo y todo esto se logra gracias al talento humano que es quien posee las habilidades, destrezas necesarias para innovar e incluso transmitir la información entre los miembros para unificar los procesos y realizar de manera eficiente las actividades.

Por lo tanto, las organizaciones inteligentes impulsa a sus miembros a compartir la información y experiencias, desarrollando todas sus habilidades, destrezas y capacidades. Gestionar el conocimiento permite mantener la producción y ser más competitivos, jugando un papel fundamental en el éxito de ese camino el liderazgo, a través del cual se pueden desarrollar las capacidades que permita guiar los cambios necesarios para adaptarse a los diversos factores del entorno. 


\section{DESARROLLO}

\section{Gestión del Conocimiento}

El factor productivo por excelencia es el conocimiento, el cual sirve de apalancamiento al creciente y veloz cambio tecnológico, el cual es definido por Arboníes (2006), como toda la información que un individuo posee en su mente, la cual es personalizada y subjetiva relacionada con hechos, procedimientos, conceptos, interpretaciones, ideas, observaciones, juicios, creencias, valores y elementos que pueden ser o no únicos, útiles, precisos o estructurales. Al respecto Toffler (1990), agrega que el conocimiento es poder y otorga la clave para producir un cambio, afirmando la superioridad del mismo por encima de los recursos monetarios y de la tecnología.

Sobre el particular Nonaka y Takeuchi (1999), afirman que el conocimiento va más allá de lo que se puede ver por encima o a simple vista (lo que está escrito), para entender lo que abarca es necesario comprenderlo como un todo incluyendo lo que está en la parte interna arduo de exteriorizar, como serían las ideas o la intuición las cuales tienen sus raíces en la parte más profunda de las acciones y la experiencia individual, así como en los ideales, valores y emociones de cada persona. Para Davenport (2001), el conocimiento es una mezcla fluida de experiencias estructuradas, valores e información que proporciona un marco para la evaluación e incorporación de nuevas experiencias e información.

Los diferentes autores, coinciden que el futuro de las organizaciones están en mano de las personas que son quienes poseen el conocimiento y con su destrezas, habilidades generan un valor económico incalculable para las organizaciones, permitiendo con el dominio de la información que las empresas obtengan una gran ventaja competitiva frente a sus competidores, aportando nuevos productos o servicios, acortando los procesos de producción con el menor tiempo de entrega y costo con la mayor cantidad de ingresos posibles, lo cual no sería posible sin ese activo intangible tan importante e indispensable para el desarrollo y permanencia en el mercado.

Según Hedlund, Forsytheb, Horvathcy Sternbergf (2003), el conocimiento es creado, transformado y difundido a toda la organización, el mismo se construye sobre la 


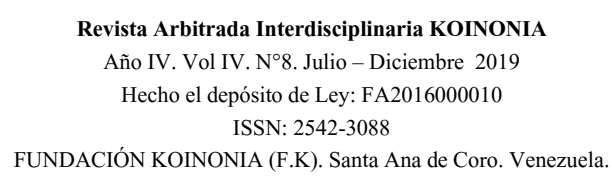

Annayeskha Grabiela González beuses

interacción entre conocimiento articulado (conocimiento explícito) y conocimiento tácito, siguiendo a Salazar (2004).El primero es un conocimiento del tipo formal y metódico que se encuentra disponible en la red de información de la empresa a disposición de los colaboradores como es el caso de los diferentes manuales de procedimientos.

Por su parte el conocimiento tácito hace referencia a esa información, destreza que posee el colaborador la cual reviste el carácter de personalísimo, con un alto nivel de dificultad para comunicar, el mismo se encuentra concentrado en el individuo y en la manera como se desenvuelve en el desempeño de sus funciones, así como influye con su desempeño en la competitividad de la organización.

En este sentido, Nonaka y Takecuchi (1995), nos habla de cuatro (4) modos de conversión del conocimiento, a través de los cuales se transfiere del individuo al grupo y a los diferentes niveles organizativos la información. (Ver figura №1)

\section{Figura $\mathrm{N}^{\circ} 1$}

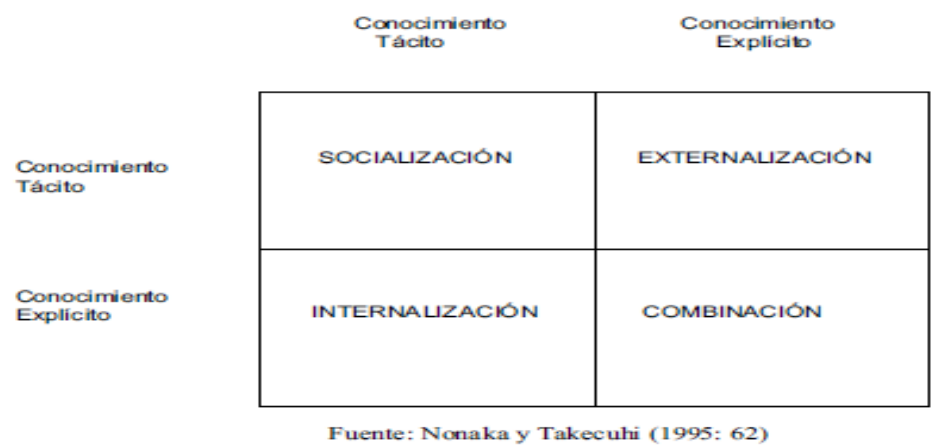

Fuente: Nonaka y Takeuchi (1995:62)

La socialización: se refiere a que las personas aprenden observando e por imitando, es decir el conocimiento tácito se transforma en tácito debido que la información transmitida no es materializada a través de manuales o instructivos impresos sino que un colaborador aprende observando cómo trabaja su compañero o al imitarlo como hacen los niños.

Externalización: el conocimiento tácito se transforma en conocimiento explicito, donde ese conocimiento que tienen los colaboradores obtenida por medios no formales o los 


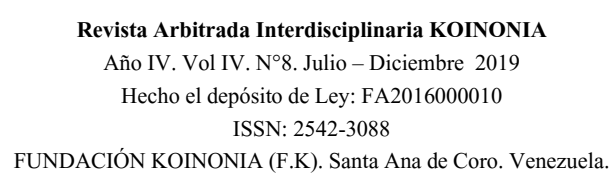

Annayeskha Grabiela González beuses

procedimientos que se realizan en la organización, es plasmada en manuales para su posterior utilización.

Interiorización: hace referencia a la conversión del conocimiento explícito en conocimiento tácito, es decir que la información plasmada en manuales, libros, instructivos, guías luego de analizada y procesada se materializa y es transmitida a través de la conducta o modelo conocido como medios no formales.

Combinación: el conocimiento explicito se transforma en explicito, como resultado de la interacción e intercambio de información, permitiendo actualizar y modificar la información, como es el caso de los colaboradores que aprenden a través de la información contemplada en los manuales y con ello pueden analizarla y transformarlo también en conocimiento explicito con el objetivo de plasmarlo y sirva como material de consulta.

Al respecto, los autores Probst, Raub y Romhardt, señalan que la Gestión del Conocimiento abarca: "funciones de planificación, organización, dirección y control, con el propósito y objetivo de adquirir, generar y potenciar los conceptos e ideas necesarias para mejorar la calidad y valor entregado a los clientes, al tiempo que se incrementa la rentabilidad financiera" (2001, p. 45). Por su parte Ramírez (2012, p.1), manifiesta que la gestión del conocimiento es "la manera cómo vamos a captar, organizar y acceder a todas las experiencias, habilidades, ideas, procesos y conocimientos que existen en una empresa y que los miembros de la organización conservan para el uso de sus funciones". Al respecto Balza (2009, p.19), manifiesta que "es la capacidad de los actores organizacionales para aprender a generar, utilizar y valorar la sabiduría humana, lo cual implica ocuparse tanto del origen como del destino del conocimiento. Según De la Calle y Ortiz (2007, p.186) "es el conjunto de procesos que utilizan el conocimiento para la identificación y explotación de los recursos intangibles existentes en la empresa así como la generación de otros nuevos".

Los mencionados autores proponen que la gestión del conocimiento, es una herramienta moderna enfocada en generar nuevo conocimiento y de organizar, procesar, aplicar el existente en las organizaciones esto con la finalidad de poder 


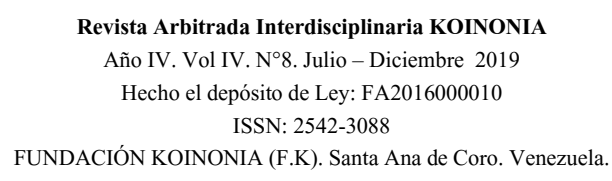

Annayeskha Grabiela González beuses

innovar y competir con otras de manera eficiente e inclusive lograr superar las costumbres, sobre todo cuando ellas impiden la normal y efectiva respuesta de la organización a los nuevos requerimientos del entorno. De allí que se considera crucial para superar los paradigmas ineficaces establecidos considerando que los mitos dificultan el proceso para encontrar respuestas a las nuevas y diversas situaciones que se presentan en la organización.

Al respecto Acasio y Colina (2016), manifiestan que la gestión del conocimiento es el resultado de la combinación entre las herramientas tecnológicas y las habilidades, destrezas, e incluso el conocimiento que poseen los talentos humanos, con la finalidad de potenciarla hasta llegar a convertirlo en una fortaleza y que se transforme en una ventaja competitividad para la organización.

De igual forma Nonaka y Takecuchi (1995), expresan que la economía del conocimiento se caracteriza por la incertidumbre y el hecho de que la ventaja competitiva se obtiene a partir de gestionar el conocimiento. Por lo tanto las organizaciones si desean permanecer y ser exitosas, deben crear constantemente un nuevo conocimiento considerando que nos desenvolvemos en mundo en constante evolución y para lograr mantenerse en el mercado es necesario innovar y transferir el conocimiento de manera eficaz y eficiente a todos los miembros de la organización e incorporarlo continuamente al proceso productivo y servicios todo esto con la finalidad de encontrar espacios de diferenciación constante.

Por lo tanto, las empresas que no logren actualizar y profesionalizar a los colaboradores van a quedar rezagadas y postergadas dado que la capacitación es uno de los pilares en el desarrollo del talento humano y para lograrlo es menester formular una política integral de personal que hará posible un crecimiento intelectual y operativo de los agentes de una organización, siendo esta la única vía para poder hacer frente a las turbulencias de los cambios antes mencionados con el personal mejor capacitado, flexible, alerta al cambio y con una nueva, clara visión del negocio.

La segunda fase es compartir, generar, descubrir conocimiento e informar a través del hacer. La última fase es la aplicación que significa transformar ese conocimiento que 
poseen los cooperadores u colaboradores en algo valioso para la organización permitiendo incrementar la productividad y ser más eficiente en concordancia con lo planteado por Barboza (2014).

\section{Modelo de Proceso de Gestión del Conocimiento}

El Modelo propuesto por Leonard Barton (1995), se refiere a las capacidades, las cuales se traducen como esa ventaja competitiva que ha adquirido la organización a lo largo de su trayectoria y que es invaluable. Para alcanzarlo es necesario tener en consideración los siguientes elementos:

\section{Se divide en tres (3) elementos:}

-Adquisición: este elemento hace referencia a generar conocimiento y explotar el contenido obtenido de la experiencia con los proyectos realizado y lo aprendido a lo largo de la vida del hombre, para ello es necesario recopilar, analizar e interpretar la información obtenida. En este sentido, Barbosa (2012), expresa que la adquisición consiste en generar, descubrir información a través del hacer.

-Selección del conocimiento: esta actividad consiste en recabar el conocimiento requerido de los colaboradores y lo presenta para materializar la actividad requerida, es decir las de adquisición, uso o interiorización. De igual forma, la selección implica otras actividades secundarias como la localización interna de la información imperiosa del conocimiento localizado y su transferencia hasta la actividad que lo necesite.

Interiorización del conocimiento: este consiste en alterar el conocimiento actual para producir uno nuevo y lograr compartir el mismo. El conocimiento original puede ser producto de la selección, adquisición o generación anterior, lo cual implicaría una serie de actividades secundarias como sería el seguimiento, los recursos del conocimiento organizativo, el entorno y la transferencia de la información .concebida para luego poder exteriorizarla, la cual consiste en emplear el conocimiento o recursos existentes para alcanzar un alto desempeño y para ello se deben emplear otras actividades como la 
seleccionar los objetivos, la producción, la transferencia del rendimiento, también se puede interiorizarla

\section{Influencia de la gestión del conocimiento}

Influencia de los Recursos: los recursos organizativos ya sean estos humanos, financieros, de conocimiento o materiales afectan la forma de llevar la gestión del conocimiento en una organización así como los recursos financieros, un ejemplo: puede ser desde el punto de vista del presupuesto y la disponibilidad financiera poner un límite a la partida destinada a las actividades de capacitación o destinadas a generar conocimiento. De igual forma cada uno de los seis (6) tipos de recursos del conocimiento puede limitar, determinar o influir en el comportamiento de la gestión del conocimiento en una organización.

Influencia de la gestión: la gestión del conocimiento no solamente se ve afectado por los diversos recursos indistintamente de cuál sea la naturaleza y por la forma como los mismos se distribuyen y son empleados. Esta influencia se emplea para determinar el estado de los recursos del conocimiento de una organización y las aptitudes de los participantes de la gestión del conocimiento así como la manipulación del mismo.

La coordinación tiene que ver con la armonización de las actividades realizadas en la organización ya que aporta los recursos adecuados en los momentos oportunos y asegura que estén correctamente relacionados entre ellos. Ejemplo: en una organización se debe contratar colaboradores con habilidades y conocimiento en las líneas estratégicas de producción y también es preciso reforzar ese conocimiento propiciando el intercambio del conocimiento entre los miembros, siendo las estructuras determinantes bajo las que se llevan a cabo la actividades de manipulación de conocimiento en circunstancias variables, la asignación de recursos financieros para dichas actividades y la cesión de participantes para las funciones de infraestructura.

Influencia del entorno: los diferentes aspectos del entorno como son la competencia, los clientes proveedores, entes gubernamentales, el mercado y el denominado clima 


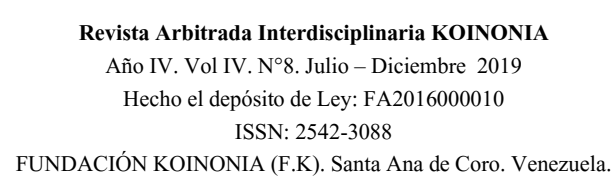

Annayeskha Grabiela González beuses

gubernamental-económico-político-social y educativo (GEPSE), como señala el modelo propuesto por Holsapple y Joshi (2002).

\section{Capital Intelectual}

Actualmente para conocer cuál es el valor del activo de una organización no puede limitarse solamente con revisar los estados financieros, es necesario considerar cual es el valor del capital intelectual que es el activo intangible más valioso con el que se cuenta hoy en día, considerando la cuantía de sus aportes y que gracias a ellos las empresas son más competitivas e innovadoras con un valor incalculable si se llega a comparar con el valor de un terreno o de una maquinaria que se va depreciando con el paso del tiempo a diferencia del capital intelectual, el cual genera un plus adicional.

De acuerdo con lo expresado por Von (2000) y Díaz (2003), al analizar el capital intelectual $(\mathrm{Cl})$ desde el contexto de la Gerencia del Conocimiento, se afirma que el $\mathrm{Cl}$ está integrado por un conjunto de activos, los cuales no se van a encontrar reflejados en el balance general o en los estados contables. Al respecto Ross y Ross (2001), afirma que los activos intangibles no son absorbidos por el balance e incluyen todo lo que está en la mente de las personas, como lo que dejan en la organización cuando se retiran.

En ese mismo orden de ideas Duarte, Jiménez y Ruiz (2007), definen el capital intelectual como: la posesión de conocimientos, experiencia aplicada, tecnología organizacional, relaciones con clientes y destrezas profesionales que dan a una empresa una ventaja competitiva. Por su parte Brooking (1997), sostiene que es una mezcla de activos inmateriales que permite funcionar a la empresa y que se dividen en cuatro (4) categorías:

-Activos de Mercado: es el potencial producto de los bienes inmateriales que guardan relación con el mercado.

-Activos de Propiedad Intelectual: Know-how encontrando en esta categoría la producción intelectual, creatividad del hombre como es la propiedad intelectual, copyright, derecho de autor, patentes. 
-Activos Centrados en el Individuo: cualificaciones que conforman al individuo hasta ser formado y transformado en lo que es hoy en día.

-Activos de Infraestructura: abarca lo relacionado con la tecnología, gestión y los procedimientos indispensables para el funcionamiento de la empresa.

Al hablar de capital intelectual es menester incluir todas las capacidades, destrezas, habilidades y el conocimiento que posee el talento humano que forma parte de la organización, abarcando también la capacidad de los líderes para aprovechar ese conocimiento y experticia individual de cada uno de los colaboradores.

En conclusión el Capital Intelectual es el conjunto de capacidades, destrezas y conocimientos del hombre los cuales generan un valor que en términos administrativos, contables y financieros no son perceptibles, pero a nivel de medición se consideran como una fortaleza con un alto nivel de impacto en los resultados organizacionales. Lo que hace posible lograr organizaciones efectivas a través de la innovación permitiendo maximizar tanto la producción como el rendimiento y disminuir los costos, alcanzando con ello ser más competitivos. Por lo tanto, se puede afirmar que una empresa es exitosa cuando busca incrementar el valor de su capital intelectual.

Por su parte Marrero (2004), manifiesta que el capital intelectual, posee tres categorías de clasificación: el capital humano, el capital estructural y el capital relacional

-Capital Humano: es el conjunto de habilidades desarrolladas como producto de la educación recibida por el individuo o la capacitación formal incluyendo en esta categoría la experiencia en las actividades tecnológicas, destrezas, actitudes y capacidades heredadas. Al respecto Stewart (2001), plantea que el conocimiento puede ser clasificado en tácito o explícito dependiendo de donde se origina el mismo y que el capital humano es interno perteneciéndole a cada persona en particular de allí que se considera la base de la generación de los otros dos tipos de Capital Intelectual.

El factor humano es un elemento calve e invaluable para la organización partiendo de la premisa que los colaboradores son quienes poseen el conocimiento indispensable para el crecimiento, desarrollo y éxito de la organización considerando que al aplicar de 
manera correcta la información que poseen coadyuva a la solución de los problemas de manera eficaz y eficiente siendo más competitivos e innovadores.

-Capital Estructural: se refiere al conocimiento que se ha capturado e institucionalizado como estructura, procesos documentados, patentes, sistema de información, manuales, archivos, bases de datos, cultura de la organización. Un capital estructural fortalecido facilita el flujo de conocimiento e implica un incremento en la eficacia, eficiencia y competitividad de la organización.

Por su parte el Capital Relacional se refiere al valor que tiene para la organización las diversas relaciones que se han concretado y se mantienen durante un período de tiempo con diferentes grupos de interés, generando valor agregado a la marca y obteniendo nuevos clientes. En este sentido siguiendo lo propuesto por Whiston (1999) se afirma que la organización al estar mejor relacionada pose a su vez mayores ventajas competitivas en virtud de su acceso oportuno a la información y al conocimiento, lo cual es determinante debido a los cambios constantes que éstos experimentan.

-Capital Cliente: hace referencia al valor obtenido por los cliente como resultado se los negociaciones realizadas por las empresas encargada de suministrar los bienes o prestar servicio con lo cual se va a mejorar la calidad del producto final ofrecido por la organización y con ello la competitividad. En este mismo sentido expone Román (2004, p.68), que "el mundo actual de los negocios exige, gente calificada, motivada, comprometida con la empresa, que apoye los planes de crecimiento".

Por lo tanto, al hablar de activo es fundamental resaltar como el de mayor valor para una empresa es el activo intangible que tiene su origen en las habilidades, actitudes, creatividad, conocimiento, destrezas de los colaboradores representando un intento sistemático y organizado para transformar la ventaja competitiva en beneficios incalculables para la organización.

En ese mismo sentido Whiston (1999); considera que si el conocimiento es poder, el aprendizaje es la clave de éste, planteamiento que refuerza la tendencia actual de 
fomentar tanto el aprendizaje individual como el organizacional para gestionar el cambio que requieren las empresas para alcanzar posiciones competitivas.

De acuerdo con los planteamientos anteriores se propone como definición de capital intelectual de una organización aquel conformado tanto por los conocimientos tácitos y explícitos de sus integrantes, la infraestructura organizacional tangible e intangible y por el nivel de relaciones interorganizacionales que promuevan la generación e intercambio de conocimientos con posibilidades reales y potenciales de ser aplicados para crear nuevos procesos, productos y servicios o mejorar los ya existentes y que incidan en el logro de beneficios sociales y económicos.

\section{Líder Gestor de Conocimiento}

Para poder implementar la Gestión del Conocimiento es necesario un líder que sea capaz de facilitar transacciones organizacionales como búsqueda y difusión de información, colaboración entre unidades y el compartir prácticas efectivas; también contribuya con tareas como la negociación, el monitoreo, la motivación y la alineación de expectativas. Fleenor (2005).

Por lo tanto, en la gestión del conocimiento el liderazgo es un factor crucial para lograr coordinar, evaluar y ser un catalizador entre las necesidades y competencias ejerciendo de esta manera un control sobre los colaboradores detectando a tiempo cuales son las necesidades, deficiencias del personal y proteger al recurso que tiene el conocimiento impulsando que sea compartido.

De acuerdo a lo antes mencionado el líder debe de conformidad con lo planteado por Holsapple y Joshi (2002) debe ser capaz de:

-Coordinar: en una organización el líder se encuentra en el deber de identificar y supervisar las actividades de manipulación del conocimiento ofreciendo un asesoramiento sobre la naturaleza de los recursos del conocimiento que son necesario para gestionar el conocimiento. De allí que la coordinación implique exigir un nivel adecuado de aptitudes de manipulación y la integración del procesamiento del conocimiento de otras operaciones organizativas. 


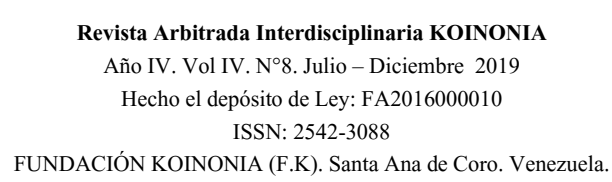

Annayeskha Grabiela González beuses

-Controlar: está relacionada con las actividades de protección, la calidad de los recursos y el procesamiento de la información, así como proteger los recursos del conocimiento de la perdida, caída en desuso, la exposición o modificación no autorizada y asimilación errónea, lo cual es menester para obtener un comportamiento eficaz de la (GC).

-Medir: implica la evaluación de los recursos del conocimiento y para ello el líder debe encargarse de coordinar, controlar el funcionamiento del procesador de conocimiento, así como la manipulación del mismo. Ofreciendo una base para lograr identificar y reconocer cuales son las actividades, recursos que aportan valor añadido, así como las áreas que presentan problemas o dificultades.

En cuanto a las perspectivas del líder en relación a la gestión del conocimiento Fleenor (2005), manifiesta que al gestionar el conocimiento se ve afectada la práctica del liderazgo de varias formas siendo una de las principales la relacionada con alcanzar las metas trazadas en el menor tiempo y costos para la organización. Considerando, que al implementar la GC se promueve la participación de un mayor número de personas que participen activamente en las acciones desarrolladas por la organización.

De allí que en virtud del conocimiento y del proceso de comunicación entre los colaboradores de una empresa es necesario contar con un líder que se encargue de ejercer las acciones necesarias para evitar que la dirección pierda el control incurriendo en la descentralización y para ello el líder tiene que ser flexible e identificar cuáles son los mecanismos más idóneos para la adaptación al cambio.

Por lo tanto el estilo del liderazgo implementado en la gestión del conocimiento debe ser claro y adecuadamente desempeñado dentro de la estructura de las organizaciones brindando un sentido acorde de dirección que apoye la gestión del conocimiento. En concordancia con lo planteado por Delgado, Pedraja y Rodríguez (2010), quienes afirman que los resultados para las fases de la gestión del conocimiento (relacionada con crear y compartir conocimiento), identificaron que el liderazgo transformacional es una determinante positiva mientras que el liderazgo transaccional es el que impacta de manera positiva y significativa la fase de aplicación del conocimiento. No existe sin 
embargo, un estilo de liderazgo determinante en la GC vista desde la búsqueda de variables explicativas en las diferentes fases de la GC, tal como lo muestran Pan y Scarbrough (1999), Politis (2001) y Rebière y Sitar (2003).

En este mismo orden de ideas Contino (2004), indica que un líder para poder gestionar de manera adecuada el conocimiento debe comprender y conocer las herramientas tecnológicas y el equipo adecuado para reducir al mínimo los errores con una gran capacidad de análisis de los datos y las habilidades en estrategias de planeación: uso apropiado de la información interna para evaluar la operación, búsqueda de soluciones y nuevas oportunidades, revisando y ajustando el plan de negocios definido.

Para Scamardo y Harnden (2007); uno de los retos para los lideres, es lograr conocer a todos sus colaboradores cuáles son las motivaciones, inquietudes, debilidades e intereses y a partir de ese conocimiento previo generar entornos que mejoren los ambientes donde se desempeñan. Al respecto el mencionado autor propone aplicar el concepto de habilidades suaves como potenciadores de desempeño del líder, sus seguidores y la organización, dentro de las cuales se han incluido los dominios de la inteligencia emocional propuestos por Goleman, los cuales se componen por la aptitud personal: la cual abarca todo lo relacionado con el autoconocimiento, autorregulación, motivación y la aptitud social donde se incluye: empatía y habilidades sociales.

Como se referenció anteriormente, dentro de las habilidades sociales se encuentra el liderazgo, conceptualizándolo dentro del contexto de la gestión del conocimiento como el individuo con la capacidad, habilidades y destrezas necesarias para lograr inspirar y guiar a grupos e individuos y que los mismos lo escuchen y lo sigan.

En virtud de lo anteriormente expuesto, se puede concluir que con desde la gestión del conocimiento se facilita la práctica del liderazgo implementada por los colaboradores dado que favorece el intercambio de información, la innovación, el desarrollo de nuevas técnicas, así como la cooperación entre los miembros de la organización, el manejo del contenido es mayor como resultado de las habilidades y destreza del personal con lo cual se facilita la implementación de las herramientas propias del liderazgo y por consiguiente impulsando el crecimiento de la empresa dado que se pueden obtener 


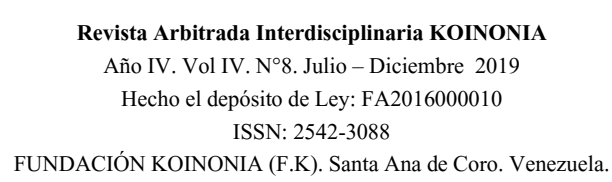

Annayeskha Grabiela González beuses

mayores beneficios y alcanzar los objetivos en el menor tiempo y costo. De acuerdo con lo expuesto por Barbosa y col. (2014).

Por su parte los mencionados autores afirman que los diferentes estilos de liderazgos afectan de diversa manera la gestión del conocimiento, de allí que se aconseja un estilo claro y precio para evitar confusión y enredos en la transmisión de la información. Por su parte Delgado, Pedraja y Rodríguez (2010), afirman que no se puede encasillar la gestión del conocimiento en un determinado tipo, aun cuando recalcan que recomiendan un liderazgo transformacional en la etapa de creación y difusión del conocimiento con la finalidad de inspirar a los colaboradores a poner en primer lugar los intereses de la empresa, logrando que transciendan los intereses personales.

Finalmente, para la etapa de implementación lo más recomendado es el liderazgo transaccional dado que este tipo de líder lo que busca es guiar a los colaboradores para que cumplan con sus actividades, ayudando a alcanzar las metas propuestas recordándoles oportunamente cuales son las tareas y las funciones de cada uno de los miembros del equipo.

De acuerdo con lo expuesto, es preciso aclarar que no existe un estilo de liderazgo ideal para manejar la gestión del conocimiento, lo importante es que el líder sepa manejar las relaciones interpersonales y conocer cuáles son las necesidades, intereses, destrezas, motivación, fortalezas de sus colaboradores para poder potenciarlas y a partir de allí emplear las estrategias necesarias lograr llevar a la organización al nivel deseado y aprovechando al máximo la gestión del conocimiento para el crecimiento de la empresa.

\section{Organización Inteligente}

Uno de los mayores retos que presentan actualmente las organizaciones es ser competitivos y para ello deben innovar logrando de esa forma mantenerse en el mercado construyendo organizaciones inteligentes, apoyándose para ello en el conocimiento que los colaboradores poseen y que una vez aplicado genera un gran 
valor económico. Por lo tanto, las organizaciones que implementan el aprendizaje permanente como partede su filosofía son conocidas como organizaciones inteligentes. En ese sentido, las organizaciones inteligentes son aquellas que tienen la capacidad organizada para tomar decisiones innovadoras sobre problemas emergentes en el mínimo tiempo posible y al menor costo, manejando recursos como la información, el conocimiento, recursos financieros y los humanos. En este orden de ideas Whiston (1999), señala que todas las organizaciones necesitan aprender y mejorar continuamente sus capacidades tomando como insumo la información generada tanto dentro como fuera de ella y procesarla para convertirla en conocimiento organizacional. De lo indicado anteriormente, puede afirmarse que las organizaciones deben ser flexibles con una estructura organizacional plana eliminando las jerarquías, facilitando los canales para el aprendizaje de los colaboradores mejorando con ello los procesos internos y su relación con el entorno, dando repuesta a los requerimientos formulados de manera rápida y eficiente demostrando adaptabilidad a los cambios del mercado.

En virtud de que el conocimiento es cambiante dependiendo del contexto en el que se encuentre y las necesidades es menester para las organizaciones evolucionar y lograr adaptarse al mercado, economía, siendo para ello elementar establecer las estrategias necesarias para superar los retos. Para esto se debe contar con un líder can las capacidades necesarias para reinventarse. Así es como Senge (2005), sostiene que una de las formas para lograr transformar a la organización y conducirlas al éxito es convirtiéndolas en organizaciones inteligentes.

Por su parte Torres y Díaz (2010); manifiestan que al hablar de organizaciones inteligentes se hace referencia a las aprendedoras considerando que se busca modificar los paradigmas y establecer una nueva forma de gestionar la empresa, la cual se mediría por la forma y la manera como logra aprender el talento humano y la organización en sí y el resultado de ese proceso se obtiene la anhelada ventaja competitiva.

En ese sentido León, Tejada \& Yataca (2003), exponen que las Organizaciones inteligentes son aquellas que aprenden permitiendo ampliar su conocimiento. Para León 
y col. (2003), una organización con capacidad de aprendizaje y de creatividad se basa en cinco (5) disciplinas elementales como son: dominio personal, trabajo en equipo, visión compartida, modelos mentales y pensamiento sistemático.

Sobre este punto en particular Wei (1999), sostiene que las organizaciones inteligentes están implantadas en equipos de alto desempeño, los cuales se unen para realizar conjuntamente el trabajo e impulsar resultados eficiente, eficaz y de alta calidad hasta que logren ser transformados en los mejores del mercado y en las cuales deben prevalecer el conocimiento tácito y explicito con el objetivo de que toda la organización se nutra con el conocimiento hasta llegar a crecer como tal.

De allí que Bravo (2010), agrega que una de las características de este tipo de organización es la preparación continua de los colaboradores, los cuales se caracterizan por ser responsables y autodisciplinados quienes siempre escuchan al cliente, identificando sus requerimientos, promoviendo el trabajo en equipo obteniendo organizaciones mucho más flexibles y planas.

De acuerdo a lo expuesto anteriormente, puede afirmarse que una organización es inteligente cuando procura el crecimiento de los colaboradores velando por la capacitación de cada uno de los miembros de su equipo y que estos apliquen ese conocimiento adquirido en la solución de los problemas e innovando transformándose en una ventaja competitiva. De allí que se apueste cada día por la preparación, desarrollo y crecimiento del talento humano que se traduce en creatividad y avance de la empresa.

Sobre este punto en particular afirma León et(al). (2003), que en las organizaciones inteligentes se encuentran dos tipos de colaboradores; los que realizan las actividades medulares de la organización los cuales se caracterizan por dirigir de manera estratégica y los colaboradores consustanciados quienes son de carácter semipermanente realizan un trabajo cotidiano y son coordinados. Por lo tanto la importancia de tener un equipo multidisciplinario para abarcar todas las áreas respondiendo a las demandas estructurales tanto interna como externa. 


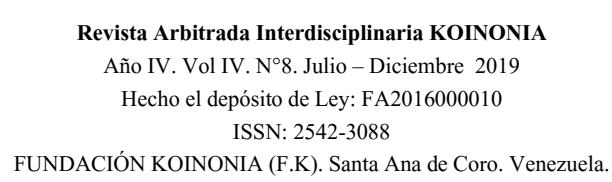

Annayeskha Grabiela González beuses

Una de las características de las organizaciones inteligentes es la flexibilidad y la libertad donde los colaboradores integran equipos de alto desempeño caracterizados por la creatividad, horarios adaptados a las necesidades del individuo quienes pueden trabajar remoto desde cualquier lugar y hora de ser necesario, empleando las herramientas y las destrezas necesarias que permita alcanzar de manera satisfactoria los objetivos trazados en el menor tiempo y costo posible permitiendo que la empresa se adapte a los nuevos requerimientos del mercado y sea. De allí que el talento humano se sienta comprometido e identificado aportando todo su conocimiento y se pueda aprovechar al máximo todo ese potencial.

En virtud de lo expuesto anteriormente se puede afirmar que las organizaciones inteligentes actualmente se presentan en cuatro (4) niveles de acuerdo con Del Carpío (2010):

-Nivel individual: se encuentra representado por el talento humano de la organización, los cuales tienen unas funciones, actividades y roles determinados, los mismos están impregnados de una cultura y comportamiento propio que va a depender del entorno donde se desenvolvieron. El reto es que el conocimiento que posee esta persona fluya a niveles más altos no se quede estancando en el hombre.

-Nivel grupal: al generar nuevo conocimiento siempre es necesario que los talentos humanos interactúen entre ellos y como producto de esa convivencia se encuentran personas con diferentes conocimientos, creencia, valores, principios e incluso con esquemas mentales diferentes con los cuales se enriquece el conocimiento individual.

-Nivel organizacional: una vez se obtienen resultados producto de la interacción entre los colaboradores de la empresa, se validan los objetivos de la misma logrando un nuevo conocimiento y con ello una ventaja competitiva única.

-Nivel interorganizacional: en este nivel se genera una conexión entre la organización con el entorno exterior donde la primera debe adaptarse a los diferentes cambios ya sean del mercado, economía o tecnológico y como resultado de esa interacción se genera un información y conocimiento relevante para el crecimiento y desarrollo de la organización. 
Por lo tanto, los líderes en las organizaciones inteligentes, según Becerra y Sánchez (2011), deben ser pilares fundamentales para enfrentar la contingencia y arbitrariedad que se les presente ante la realidad cambiante. El líder siempre debe mostrar cuales son las estrategias a seguir, adaptándose a las situaciones de cambio.

Por su parte manifiesta Morín (2005), que los líderes de las organizaciones inteligentes son unos maestros encargados de diseñar, modelar, administrar y guiar las organizaciones con el compromiso de incrementar la capacidad para la poder entender, analizar desde todas las aristas los modelos mentales, permitiendo un crecimiento y aprendizaje.

Para Senge (2005), una de las características principales de las organizaciones inteligentes es que son menos jerárquicas convirtiéndose en horizontales o en red con la finalidad de ser más eficiente donde cada uno de los integrantes participa en la toma de decisiones, con un alto grado de autonomía y el poder es descentralizado en manos de los cooperadores.

\section{Modelo Organización Inteligente}

Como puede observarse en el modelo de organizaciones inteligente se emplean tres (3) procesos que permiten procesar e integrar la información y el conocimiento para actuar de forma inteligente como son: la comprensión del entorno tanto interno como externo de la organización para lograr generar conocimiento nuevo y finalmente generar la decisión que no es más que la forma como la empresa procesa la información para resolver la incertidumbre.

En las organizaciones inteligentes es fundamental trabajar en equipo considerando que para transmitir la información y lograr una retroalimentación es necesario compartir con otros individuos evaluando que el proceso de aprendizaje es individual y depende de cada persona, el cual abarca no solo adquirir nuevo conocimiento sino un cambio de paradigma, perspectiva o enfoque.

Para que una organización sea inteligente según lo expuesto de Senge (2005), es necesario desarrollar cinco (5) competencias básicas entre las que se encuentran el 


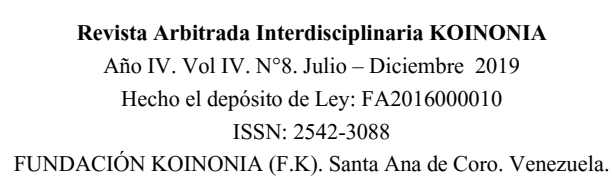

Annayeskha Grabiela González beuses

pensamiento sistémico: que es la actitud del individuo basada en su percepción preconcebida del mundo; los modelos mentales lo cual hace referencia a las concepciones del hombre sus creencias, esquemas; el aprendizaje en equipo: para intercambiar información y transmitirla es necesario un grupo de individuos que interactúen entre sí; el dominio personal: se refiere al trabajo interno que debe realizar el individuo para lograr comprender la información; y finalmente la visión compartida: para alcanzarla es necesario que la visión organizacional se compagine con la del colaborador.

\section{Metodología}

El tipo de investigación es analítica documental. Según Chávez (2007), un estudio se considera documental cuando la finalidad de la investigación es obtener información partiendo de los documentos previamente escritos, los cuales pueden ser analizados. El diseño de la investigación es de tipo bibliográfico no experimental, transeccional, puesto que los datos se analizaron e interpretaron en un solo momento, la población es netamente documental.

La técnica empleada para realizar el análisis e interpretación de los datos obtenidos es la técnica cualitativa de análisis crítico. Dado que se utilizaron investigaciones previas que recogen las últimas innovaciones plasmadas en material bibliográfico como son: libros, artículos científicos y consultas realizadas en línea

Al respecto el autor Arias (2012: 25), plantea que se entiende por investigación documental aquella cuyo proceso se encuentra basado en la búsqueda, recuperación, análisis, crítica e interpretación de datos secundarios, es decir, los obtenidos y registrados con anterioridad por otros investigadores en fuentes documentales: impresas, audiovisuales o electrónicas.

En ese sentido, el diseño de la investigación es de tipo bibliográfico no experimental. De allí que Sabino (2002), plantea que el diseño de tipo bibliográfico no experimental, se refiere a que los datos e información empleados proceden de documentos escritos. En el mismo orden de ideas, Palella y Martins (2006), expone que se está en presencia 
de una investigación no experimental, cuando se realiza sin manipular de manera deliberada las variables objeto de estudio.

\section{CONCLUSIONES}

Tanto los cambios del mercado como la globalización han generado una diversidad de cambios tanto en los procesos productivos, como en los intereses y las necesidades del consumidor, en la estructura de la organización y en la concepción del trabajador, donde el capital intelectual se convierte en el activo intangible de mayor valor siendo indispensable para el desarrollo, logrando que la organización sea mucho más rentable e innovadora.

Desde esta óptica se desarrolla la gerencia del conocimiento, modelo que permite disponer del capital intelectual del talento humano de la organización, donde el conocimiento es transferido de manera tacita o explicita entre los colaboradores con la finalidad de que todos puedan hacer uso del mismo y ser más productivos.

Por lo tanto, el conocimiento es el recurso de mayor valor con el que cuentan las organizaciones, el cual es ilimitado, flexible y cambiante, de allí que las destrezas, habilidades e incluso la información que posee una persona debe ir cambiando periódicamente para lograr adaptarse dependiendo de las experiencias y el contexto donde se desarrolle o desenvuelva el individuo. Por lo tanto el capital intelectual, es el vehículo a través del cual se aportan soluciones a los problemas generando con ello ventajas competitivas obteniendo empresas innovadoras y mucho más productivas considerando que el conocimiento es un recurso invaluable. Convirtiéndose en una pieza clave para el desarrollo y este lo poseen lo individuos quienes pasan de ser sujetos pasivos a activos en virtud del aporte de los mismos.

En virtud de las exigencias del mercado así como de la competitividad entre las organizaciones es fundamental para las empresas contar con colaboradores dotados de habilidades, destrezas y el conocimiento necesario para que con esos aportes las mismas puedan ser sostenibles, innovadoras, competitivas y mantenerse a lo largo del tiempo. Para ello es indispensable que el departamento de gestión del talento de las 
organizaciones se enfoque en la gerencia del conocimiento para promover, impulsar las destrezas, habilidades e información entre los colaboradores que hacen vida, garantizando la transmisión del conocimiento entre los miembros.

En virtud de los expuesto anteriormente se puede afirmar que el éxito en la implementación de la Gestión del Conocimiento requiere de líderes dotados de inteligencia lingüística e interpersonal para lograr el intercambio de información y desarrollar el criterio de organización inteligente, considerando que es el responsable de iniciar, impulsar y coordinar los procesos de gestión del conocimiento, con el fin de maximizar la creación, el descubrimiento y la diseminación del conocimiento organizacional.

Por lo tanto, el capital intelectual concadenado con la gestión del conocimiento son necesarios para la construcción de organizaciones inteligentes donde cada uno de los colaboradores son importantes y la toma de decisión tiene un elevado grado de autonomía, encontrándose el poder descentralizado, es decir distribuido a lo largo de la estructura de la organización y no solo en manos de la junta directiva.

En virtud de lo expuesto anteriormente, puede afirmar que en las organizaciones inteligentes es fundamental contar con un talento humano dotado de habilidades y destrezas necesarias para lograr innovar y como resultado de ello obtener una ventaja competitiva en el mercado generando organizaciones mucho más rentables e incluso sostenibles. Para ello, es necesario contar con un líder que logre potenciar al máximo a sus colaboradores, impulsando el conocimiento y la transferencia del mismo entre los diferentes miembros del equipo con el objetivo de mejorar los procesos productivos, ser más competitivos y alcanzar las metas trazadas con el menor costo/tiempo posible. Siendo fundamental que el líder conozca las debilidades y fortalezas de cada colaborador. 


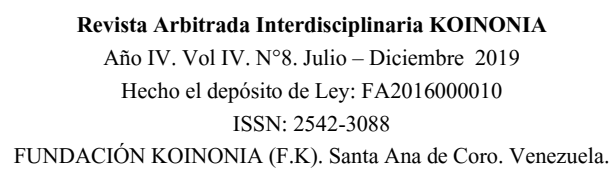

Annayeskha Grabiela González beuses

\section{REFERENCIAS CONSULTADAS}

1. Acasio, F. y Colina, F. (2016). La investigación desde la gestión del conocimiento en el contexto de las universidades nacionales experimentales. Revista Arbitrada Interdisciplinaria de Ciencias de la Educación, Turismo, Ciencias Sociales y Económica, Ciencia del Agro y Mar y Ciencias Exactas y Aplicadas.(KOINONIA). Coro. Venezuela, I (1), 88-100

2. Arboníes, Á. (2006). Conocimiento para innovar. España. Editorial Díaz de Santos.

3. Arias, F. (2012). El proyecto de investigación. Introducción a la metodología científica. Sexta Edición. Caracas: Editorial Espíteme, C.A

4. Balza, A. (2009). Gestión del conocimiento, tecnologías de la información y aprendizaje organizacional. Venezuela: Universidad Nacional Experimental Simón Rodríguez, Consejo de Desarrollo Científico Humanístico y Tecnológico (CDCHT).

5. Barbosa, D. (2012). La gestión del conocimiento desde la perspectiva de la dirección estratégica de recursos humanos. Bogotá. Editorial Universidad del Rosario.

6. Barboza, D., Ramírez, A., y Hidalgo, A., (2014). Gestión del conocimiento y liderazgo: perspectivas de relación. Revistas Científicas de América Latina y el Caribe Diversitas. 1 (10), 057-070.

7. Barton, L. (1995). Wellsprings of Knowledge: Building and Sustaining the sources of Innovation. Harvard Business Scholl Press.

8. Becerra, M., y Sánchez, L. (2011). El liderazgo en las organizaciones inteligentes. Revista Científica del Centro de Investigación y Estudios Generales. Barquisimeto. Venezuela, 1(4), 61-71

9. Bravo, B. (2010). Sistemas Avanzados de Gestión. Organizaciones Inteligentes. Cali. Colombia. Universidad de Buenaventura.

10. Brooking, A. (1997). El Capital Intelectual. Barcelona. Paidos Empresas.

11. Chávez, N. (2007).Introducción a la Investigación Educativa. (2 ${ }^{\mathrm{a}}$ Ed.). Maracaibo: Universal.

12. Contino, D. (2004). Leadership Competencies: Knowledge, Skills, and Aptitudes Nurses Need to Lead Organizations Effectively. Critica Nurse Care, 24(3), 52-64.

13. Davenport, T. (2001).Knowledge and the future of management. En Bennis G. M. Spreitzer, W. G. y Cummings T. G. (Eds.).

14. Del Carpío, I. (2010). Modelo de Organización Inteligente basado en el Enfoque CRM: Caso UNI - FIIS, (2010). Tesis. Lima - Perú.

15. De la Calle, C., y Urbina, M. (2007). Los Modelos de Capital Intelectual en las Empresas Españolas: Una Aplicación Práctica. Facultad de Ciencias Jurídicas y Sociales de la Universidad Rey Juan Carlos. Madrid España.

16. Delgado. M., Pedraja, L., y Rodríguez, E. (2010). Gestión del conocimiento, liderazgo, diseño e implementación de la estrategia: un estudio empírico en pequeñas y medianas empresas, 18(3), 373-282. 


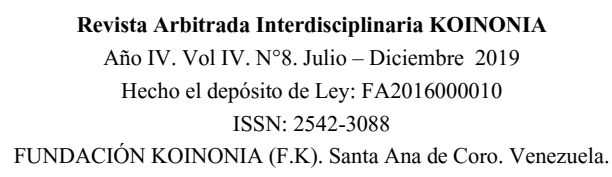

Annayeskha Grabiela González beuses

17. Díaz, R (2003). Activos Intangibles: Tecnología, Gerencia y Sociedad. Bogotá, Colombia: Ediciones Mundo Organizacional.

18. Fleenor, J. (2005). A question of leadership. Leadership in Action, 25(3), 14.

19. Hedlund, J., Forsytheb, J., Horvathc, A., Williams, W., Sternbergf, R. (2003). Identifying and assessing tacit Knowledge: understanding the practical intelligence of military leaders. The leadership Quaterly, 14, 117-140.

20. Hsieh, T. (2011). How Zappos Creates Happy Customers and Employee. México. Great Place to Work.

21. Holsapple, C., y Joshi, K. (2002). Knowledge Management: A Threefold. Washington. Usa. Framework. Taylor \& Francis.

22. León, R., Tejada, E. \& Yataca, M. (2003). Las organizaciones inteligentes. Industrial Data. Notificación Científica. Universidad Nacional Mayor de San Marcos. Lima.

23. Marrero, I. (2004). La Gestión de Información y la Gestión del Conocimiento del siglo XXI, puntos en contacto, analogías y divergencias. Tesis de Diploma. Facultad de Comunicación Social y Ciencias de la Información. Universidad de La Habana. Cuba.

24. Morín, E. (2005). Introducción al pensamiento complejo. Barcelona. España Gedisa Editorial.

25. Nonaka, I., y Takeuchi, H. (1995). The knowledge-creating company: How Japanese companies create the Dynamics of Innovation, Oxford University Press. New York.

26. Nonaka, I., y Takeuchi, H. (1999). La Organización creadora del conocimiento. México. Primera edición en español.

27. Palella, S. y Martins F. (2006). Metodología de la investigación cuantitativa. Segunda edición. Caracas: FEDUPEL.

28. Pan, S., Scarbrough, H. (1999).Knowledge management in practice: An exploratory cas study. Technology Analysis and Strategic Management.

29. Politis, J. (2001). The relationship of various leadership styles to knowledge management, Leadership Organizational Development Journal. doi: 10.1057/palgrave.kmrp.8500004.

30. Probst, G., Raud, S. y Romhaedt K. (2001). Management del Conocimiento: Una Gestión Participativa. Barcelona, España. Ediciones Gestión 3000.

31. Ramírez, G. (2012). La gestión del conocimiento como generadora de ventaja competitiva. Trabajo de Grado. Facultad de Ciencias Administrativas. Universidad del Rosario.

32. Rebière, V., y Sitar, S. (2003).Critical role of leadership in nurturing a knowledgesupporting culture. Knowledge Management Researched and Practice, 1, 39-48

33. Ross, J., Ross, G. (2001). Capital Intelectual: el valor intangible de la empresa. Barcelona: Ediciones Paidós Ibérica. S.A.

34. Sabino, C. (2002). ¿Cómo hacer una Tesis? Panapo. Caracas: Editorial. 


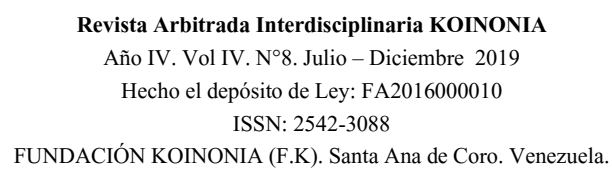

Annayeskha Grabiela González beuses

35. Scamardo, M. y Harnden, S. (2007). A Manager Coaching Group Model: Applying Leadership Knowledge. Journal of Workplace Behavioral Health, 22(2-3), 127143.doi: 10.1300/J490v22n02_09.

36. Senge, P. (2005). La Quinta Disciplina: Como Impulsar el Aprendizaje en la Organización Inteligente. Barcelona España. $2^{\circ}$ edición.

37. Stewart, T. (2001). The Brainpower. Clásicos de Revistas. Gestión del Conocimiento. Recuperado el 15 noviembre de 2018 de http://derevistas.com/contenido/artículo.php

38. Toffler, A. (1990). Powershift: knowledge, wealth and violence at the edge of the $21^{\text {st }}$ century. New York. Bantam Books.

39. Torres, L., y Díaz, J. (2010). Organizaciones Inteligentes Basadas en Inteligencia: Aproximación a una Realidad Empresarial del Siglo XXI. Revista Científica Teorías, Enfoques y Aplicaciones en las Ciencias Sociales, 03(05), 117-127.

40. Von, J. (2000). Capital Intelectual: Modelos de Gerencia en Investigación y Desarrollo. Revista Espacios. (12).

41. Whiston, T. (1999). La organización que aprende en: Manual de Gestión en Tecnología, Tomo I. Colombia. McGraw-Hill Interamericana, S.A.

42. Wei, C (1999). La organización inteligente: el empleo de la información para dar significado, crear conocimiento y tomar decisiones. México, Oxford University Press.

\section{REFERENCES CONSULTED}

1. Acasio, F. y Colina, F. (2016). Research from knowledge management in the context of national experimental universities, and interdisciplinary peer-review journal in the sciences and economics, agriculture and sea, science, and exact and applied sciences. (KOINONIA). Coro. Venezuela, I (1), 88-100

2. Arboníes, Á. (2006). Knowledge to innovate. Spain. Editorial Díaz de Santos.

3. Arias, F. (2012). The research project. Introduction to scientific methodology. Sixth edition. Caracas: Editorial Espíteme, C.A

4. Balza, A. (2009). Knowledge management, information technologies and organizational learning. Venezuela: Simón Rodríguez National Experimental University, Council of Humanistic and Technological Scientific Development (CDCHT).

5. Barbosa, D. (2012). Knowledge management from the perspective of the strategic direction of human resources. Bogotá Editorial University of Rosario.

6. Barboza, D., Ramírez, A., and Hidalgo, A., (2014). Knowledge management and leadership: relationship perspectives. Scientific Journals of Latin America and the Caribbean Diversitas.1 (10), 057-070.

7. Barton, L. (1995). Wellsprings of Knowledge: Building and Sustaining the sources of Innovation. Harvard Business Scholl Press. 


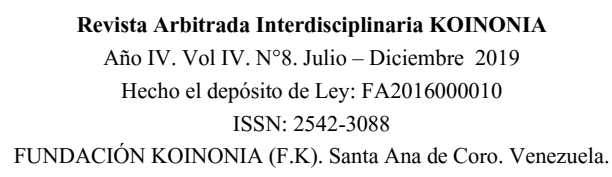

Annayeskha Grabiela González beuses

8. Becerra, M., and Sánchez, L. (2011). Leadership in smart organizations. Scientific Journal of the Research and General Studies Center. Barquisimeto. Venezuela, 1 (4), 61-71

9. Bravo, B. (2010). Advanced Management Systems. Smart Organizations Cali. Colombia. University of Buenaventura.

10. Brooking, A. (1997). The Intellectual Capital. Barcelona. Companies Paidos.

11. Chávez, N. (2007) .Introduction to Educational Research. (2nd Ed.). Maracaibo: Universal.

12. Contino, D. (2004). Leadership Competencies: Knowledge, Skills, and Skills Nurses Need to Lead Organizations Effectively. Critica Nurse Care, 24 (3), 52-64.

13. Davenport, T. (2001). Knowledge and the future of management. In Bennis G. M. Spreitzer, W. G. and Cummings T. G. (Eds.).

14. Del Carpío, I. (2010). Intelligent Organization Model based on the CRM Approach: Case UNI - FIIS, (2010). Thesis. Lima Peru.

15. De la Calle, C., and Urbina, M. (2007). The Models of Intellectual Capital in Spanish Companies: A Practical Application. Faculty of Legal and Social Sciences of the Rey Juan Carlos University. Madrid Spain.

16. Delgado. M., Pedraja, L., and Rodríguez, E. (2010). Knowledge management, leadership, design and implementation of the strategy: an empirical study in small and medium enterprises, 18 (3), 373-282.

17. Díaz, R (2003). Intangible Assets: Technology, Management and Society. Bogotá, Colombia: World Organizational Editions.

18. Fleenor, J. (2005). A question of leadership. Leadership in Action, 25 (3), 14.

19. Hedlund, J., Forsytheb, J., Horvathc, A., Williams, W., Sternbergf, R. (2003). Identifying and assessing tacit Knowledge: understanding the practical intelligence of military leaders. The leadership Quaterly, 14, 117-140.

20. Hsieh, T. (2011). How Zappos Creates Happy Customers and Employee. Mexico. Great Place to Work.

21. Holsapple, C., and Joshi, K. (2002). Knowledge Management: A Threefold. Washington. Uses. Framework Taylor \& Francis.

22. León, R., Tejada, E. \& Yataca, M. (2003). Smart organizations Industrial Data Scientific Notification National University of San Marcos. Lime.

23. Marrero, I. (2004). Information Management and Knowledge Management of the 21 st century, points in contact, analogies and divergences. Diploma Thesis. School of Social Communication and Information Sciences. University of Havana. Cuba.

24. Morín, E. (2005). Introduction to complex thinking. Barcelona. Spain Gedisa Editorial

25. Nonaka, I., and Takeuchi, H. (1995). The knowledge-creating company: How Japanese companies create the Dynamics of Innovation, Oxford University Press. New York

26. Nonaka, I., and Takeuchi, H. (1999). The Organization that created knowledge. Mexico. First edition in Spanish. 


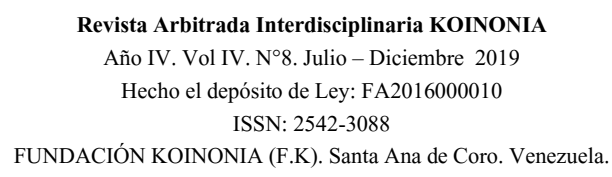

Annayeskha Grabiela González beuses

27.Palella, S. and Martins F. (2006). Methodology of quantitative research. Second edition. Caracas: FEDUPEL.

28. Pan, S., Scarbrough, H. (1999). Knowledge management in practice: An exploratory cas study. Technology Analysis and Strategic Management.

29. Politis, J. (2001). The relationship of various leadership styles to knowledge management, Leadership Organizational Development Journal. doi: 10.1057/ palgrave.kmrp.8500004.

30. Probst, G., Raud, S. and Romhaedt K. (2001). Management of Knowledge: A Participatory Management. Barcelona, Spain. Editions Management 3000.

31. Ramírez, G. (2012). Knowledge management as a generator of competitive advantage. Degree work. Faculty of Administrative Sciences. University of Rosario.

32. Rebière, V., and Sitar, S. (2003). Critical role of leadership in nurturing a knowledge- supporting culture. Knowledge Management Researched and Practice, 1, 39-48

33. Ross, J., Ross, G. (2001). Intellectual Capital: the intangible value of the company. Barcelona: Editions Paidos Ibérica. S.A.

34. Sabino, C. (2002). How to do a Thesis? Panapo. Caracas: Editorial.

35. Scamardo, M. and Harnden, S. (2007). A Manager Coaching Group Model: Applying Leadership Knowledge. Journal of Workplace Behavioral Health, 22 (23), 127-143. doi: 10.1300 / J490v22n02_09.

36. Senge, P. (2005). The Fifth Discipline: How to Promote Learning in the Intelligent Organization. Barcelona Spain 2nd edition.

37. Stewart, T. (2001). The Brainpower. Classics of Magazines. Knowledge Management Retrieved on November 15, 2018 from http://derevistas.com/contenido/artículo.php

38. Toffler, A. (1990). Powershift: knowledge, wealth and violence at the edge of the 21st century. New York Bantam Books.

39. Torres, L., and Diaz, J. (2010). Intelligent Organizations Based on Intelligence: Approach to a Business Reality of the XXI Century. Scientific Magazine Theories, Approaches and Applications in the Social Sciences, 03 (05), 117-127.

40. Von, J. (2000). Intellectual Capital: Management Models in Research and Development. Spacious Magazine. (12).

41. Whiston, T. (1999). The organization that learns in: Technology Management Manual, Volume I. Colombia. McGraw-Hill Interamericana, S.A.

42. Wei, C (1999). The intelligent organization: the use of information to give meaning, create knowledge and make decisions. Mexico, Oxford University Press.

(C2019 por el autor. Este artículo es de acceso abierto y distribuido según los términos y condiciones de la licencia Creative Commons Atribución-NoComercial-Compartirlgual 4.0 Internacional (CC BY-NC-SA 4.0) (https://creativecommons.org/licenses/by-nc-sa/4.0/). 